Article

\title{
Rapid Sampling of Escherichia coli After Changing Oxygen Conditions Reveals Transcriptional Dynamics
}

\author{
Joachim von Wulffen ${ }^{1}$, Andreas Ulmer ${ }^{1}$, Günter Jäger ${ }^{2}$, Oliver Sawodny ${ }^{1, *}$ and Ronny Feuer ${ }^{1}$ \\ 1 Institute for System Dynamics, University of Stuttgart, Keplerstraße 7, 70174 Stuttgart, Germany; \\ vonwulffen@isys.uni-stuttgart.de (J.W.); ulmer.bio@gmail.com (A.U.); \\ ronny.feuer@isys.uni-stuttgart.de (R.F.) \\ 2 Insitute of Medical Genetics and Applied Genomics, University of Tübingen, Geschwister-Scholl-Platz, \\ 72074 Tübingen, Germany; guenter.jaeger@cegat.de \\ * Correspondence: oliver.sawodny@isys.uni-stuttgart.de; Tel.: +49-711-685-66302
}

Academic Editor: Thierry Wirth

Received: 15 December 2016; Accepted: 20 February 2017; Published: 28 February 2017

\begin{abstract}
Escherichia coli is able to shift between anaerobic and aerobic metabolism by adapting its gene expression, e.g., of metabolic genes, to the new environment. The dynamics of gene expression that result from environmental shifts are limited, amongst others, by the time needed for regulation and transcription elongation. In this study, we examined gene expression dynamics after an anaerobic-to-aerobic shift on a short time scale $(0.5,1,2,5$, and $10 \mathrm{~min})$ by RNA sequencing with emphasis on delay times and transcriptional elongation rates (TER). Transient expression patterns and timing of differential expression, characterized by delay and elongation, were identified as key features of the dataset. Gene ontology enrichment analysis revealed early upregulation of respiratory and iron-related gene sets. We inferred specific TERs of 89 operons with a mean TER of $42.0 \mathrm{nt} / \mathrm{s}$ and mean delay time of $22.4 \mathrm{~s}$. TERs correlate with sequence features, such as codon bias, whereas delay times correlate with the involvement of regulators. The presented data illustrate that at very short times after a shift in oxygenation, extensional changes of the transcriptome, such as temporary responses, can be observed. Besides regulation, TERs contribute to the dynamics of gene expression.
\end{abstract}

Keywords: transcriptional elongation rates; RNA sequencing; transition; oxygen; Escherichia coli

\section{Introduction}

Escherichia coli is able to adapt to various changes in environmental conditions. An important determinant of growth is the presence of extracellular oxygen. Oxygen availability changes in the natural niches of E. coli, as well as in biotechnological settings. In industrial scale continuously stirred tank reactors (CSTR) inhomogeneities of available oxygen arise from insufficient mixing compared to the laboratory scale. Mixing times in large CSTRs are in the range of $2 \mathrm{~min}$ or more ( $140 \mathrm{~s}$ for a 120,000-L reactor [1]) while oxygen depletion can occur in less than a minute [2]. Therefore, cells circulating through the CSTR volume experience a constantly changing oxygen supply, necessitating constant adaptation of the cells to the respective conditions. Inhomogeneities of the dissolved oxygen are presumed to be an important cause for yield losses after upscaling [2,3]. In this study, we analyze the early transcriptional responses of an anaerobic $E$. coli batch culture to the onset of aeration by RNA sequencing.

Previous studies on transcriptional responses to an aerobic shift (from anaerobic to aerobic conditions) considered chemostat cultivations and longer time scales only (5-60 $\mathrm{min}$ [4], 2-20 $\mathrm{min}$ [5], and 5-120 $\mathrm{min}[6])$, or only moderate shifts in oxygen supply [6,7]. These studies were mostly motivated 
by the naturally occurring transition between anaerobic (in the host's intestines) and aerobic (outside the host) conditions. Here, we focus on fast transcriptomic changes of a batch culture in response to an aerobic shift using RNA sequencing.

Although other substrates are also inhomogeneously distributed in large scale cultivations, oxygen has a crucial role. Firstly, oxygen is not a prerequisite for growth, as E. coli cells grow anaerobically as well, but with a lower rate. However, switching from anaerobic to aerobic metabolism requires extensive adjustments of the cellular proteome and, as a precondition, of the transcriptome. This might even include the expression of genes that code for suboptimally efficient enzymes if their expression is faster than their more efficient alternatives [8]. Secondly, exposure to oxygen can cause excessive generation of reactive oxygen species (ROS), which provoke cellular stress. On the transcriptomic level, expression of stress genes can be seen indicative of present stress conditions [9].

Gene expression is, in terms of energy consumption, a costly undertaking considering limited cellular resources, as demonstrated in a setting of fluctuating carbon and nitrogen supply $[10,11]$. Therefore, tight regulation is needed to avoid unnecessary gene expression and assure optimal adaptation considering evolutionary pressure [12].

Another aspect demonstrating the need for basal expression of yet unutilized genes is the response time elapsing from the occurrence of the environmental shift to increased levels of functional protein. There are several phases that need to pass before the newly synthesized protein becomes active, including sensing and regulation of transcriptional initiation (delay time), transcription elongation, and translation, as well as post-translational modifications. The first two of these, delay time and transcription, can be approached with time series RNA sequencing measurements. Using rifampicin, an inhibitor of transcriptional initiation, operon-specific transcriptional elongation rates (TERs) have been inferred [13]. Using oxygen shift as a trigger for gene expression changes, we were able to derive delay times and TERs and compare them with literature values.

Translation in E. coli can occur cotranscriptionally [14], and it has been postulated that ribosomes and RNA polymerases cooperatively determine elongation rates $[15,16]$ and suppress termination (antitermination [17-19]). Therefore, TERs are valuable for estimating the minimal amount of time required to synthesize new proteins after environmental shift.

\section{Materials and Methods}

\subsection{Strain and Growth Conditions}

E. coli K-12 strain W3110 [20,21] was grown anaerobically in defined medium (Table S1) at pH7 and $37^{\circ} \mathrm{C}$ stirred at $500 \mathrm{rpm}$ with a Rushton turbine in a 3-liter bioreactor until the culture reached an optical density at $600 \mathrm{~nm}\left(\mathrm{OD}_{600 \mathrm{~nm}}\right)$ of 3 . At that point, the first sample was drawn and air supply was initiated subsequently at $1 \mathrm{~L} / \mathrm{min}$. Additional samples were taken at $0.5,1,2,5$, and 10 min after the onset of aeration and treated immediately with RNAprotect Cell Reagent (Qiagen, Hilden, Germany) according to manufacturer's instructions. Cell pellets were stored at $-80^{\circ} \mathrm{C}$ until preparation. The experiment was repeated in three biological replicates.

\subsection{RNA Sequencing}

Ribosomal RNA was removed from transcripts of RNA protected samples using RiboZero rRNA Removal Kit (Illumina, San Diego, CA, USA). Sequencing libraries were prepared with TruSeq RNA Sample Preparation Kit v2 (Illumina). The quality of RNA preparations was assessed electrophoretically with Agilent BioAnalyzer (Agilent, Santa Clara, CA, USA). Equimolar amounts of all libraries were pooled and loaded onto an Illumina GAIIx flow cell and bound molecules were clonally amplified on a cBot instrument (Illumina). Subsequently, RNA fragments were sequenced on an Illumina HiSeq 2500. The quality of the sequencing was assessed using FastQC [22].

Obtained sequences were aligned against the reference genome NC007779.1 [23] using the tool STAR [24]. Gene counting was performed with HTSeq [25], filtering out overlapping regions. The 
obtained data have been deposited in the National Center for Biotechnology Information (NCBI)'s Gene Expression Omnibus (GEO) [26] and are accessible through GEO Series accession number GSE71562. The Bioconductor package edgeR $[27,28]$ was applied for statistical analysis. Total mapped read counts ranged from 5.6 million to 11.0 million. Read counts were transformed to counts per million taking into account the different library sizes. Normalization with the respective gene lengths yields reads per kilobase per million reads (RPKM), which are used for plotting. A negative binomial model was fitted with the count data and the common and tag-wise dispersions were estimated. Subsequently, a generalized linear model was fitted to the data and $p$ values were calculated. The resulting $p$ values were corrected for multiple testing using the false discovery rate (FDR) method [29] (data available in Table S2). Genes with FDR corrected $p$ values of less than 0.05 and absolute fold changes greater than 2 were assumed as differentially expressed (DE). Venn diagrams were generated with the VennDiagram R package [30].

\subsection{Principal Component Analysis}

The regularized logarithm transformation of the Bioconductor package DESeq2 was applied to the count data to make the data more homoscedastic [31]. This approach balances the high counts of highly expressed genes by taking the logarithm with the large fold changes (FC) of weakly expressed genes by shrinking. These transformed count numbers were used for principal component analysis (PCA) employing the prcomp () command in R [28,32].

\subsection{Regulon and Gene Ontology Enrichments}

For each time point, genes with an absolute fold change greater than 2 and FDR-corrected $p$ values of less than 0.05 were filtered and sorted for up- or downregulation. Enrichment of regulons and gene ontology (GO) classes (biological process) were analyzed using the smart tables functions of Pathway Tools [33,34] with Bonferroni correction.

\subsection{TER Estimation}

As in [13], operon sequences were partitioned into 300-nt bins and counts were generated for each bin using the R package GenomicAlignments [35]. RPKM values were derived from counts as described above and subsequently fitted to the differential equation

$$
\frac{d\left[m R N A_{i}\right]}{d t}=s_{b, i}+\Theta\left(t-T_{d, i}\right) \cdot s_{a, i}-\left(\mu+\gamma_{i}\right) \cdot\left[m R N A_{i}\right](t)
$$

with square brackets denoting concentrations in RPKM, $s_{b, i}$ basal expression rate, $s_{a, i}$ activated expression rate, $T_{d, i}$ the delay time until differential expression sets in, $\mu$ the growth rate and $\gamma_{i}$ degradation rate of the mRNA of gene $i$. The number of measurements included in each bin's regression had to be adjusted manually in order to estimate the initial adaptation with one change of expression rates, only (i.e., from $s_{b, i}$ to $\left(s_{b, i}+s_{a, i}\right)$ or from $\left(s_{b, i}+s_{a, i}\right)$ to $\left.s_{b, i}\right)$. For example, the $c y d A B$ operon, illustrated in Figure 5a,b, is transiently upregulated. For the bins 0 to 4 , only the first three time points were included in the parameter fit, since expression increases and decreases fast. Parameter estimation of the subsequent bins 5-8 was performed on the initial four time points, since the increased expression was delayed. Where possible, $\gamma_{i}$ was taken from the literature [36], if not it was fitted to the data. $\Theta$ is a step function and is defined for cases with increasing expression as:

$$
\Theta_{\text {incr }}(t)= \begin{cases}0 & t<0 \\ 1 & t \geq 0\end{cases}
$$

and in cases with decreasing expression as $\Theta_{\text {decr }}(t)=1-\Theta_{\text {incr }}(t)$. Numerical parameter estimation was performed using Mathematica 10 (Wolfram, Oxfordshire, UK) using the simulated annealing algorithm [37] with the least squares estimator. Linear regression was applied to estimate the slope of 
the delay times $T_{d}$ over the mean bin positions weighted by the $95 \%$ confidence intervals of the $T_{d}$. The inverse of the estimated slope represents the average TER. The intersection of the regression curve with the $0 \mathrm{nt}$ position of the operon gives the overall delay time of the operon (illustrated in Figure $5 \mathrm{a}, \mathrm{b}$ ).

The codon adaptation index (CAI) was calculated from the operon sequences as described in [38] using the codon frequency table from the Kazusa Codon Usage Database [39]. The index of translation elongation (ITE) was calculated as stated in [40] using the provided data set. Folding energies were calculated from sequences using the ViennaRNA package [41,42]. The regulatory and sigma factor networks were taken from the EcoCyc E. coli Database [43].

\section{Results}

E. coli K-12 W3110 was cultured anaerobically in a laboratory-scale CSTR. At a predefined point, aeration was initiated. Subsequently, samples were taken within $10 \mathrm{~min}$ after the onset of aeration (a time course of the dissolved oxygen concentration is provided in Figure S1). The mRNA of all samples were sequenced and evaluated statistically (Table S2, raw data are available from NCBI GEO, accession number GSE71562).

\subsection{General Properties of the Dataset}

\subsubsection{Differentially Expressed Genes}

Figure 1 shows the number of statistically significant up- and downregulated genes for each time point. Genes with absolute FC greater than 2 and FDR corrected $p$ values lower than 0.05 were considered DE. The maximal difference is reached at $10 \mathrm{~min}$ where $7.2 \%$ of the genome is upregulated and $5.7 \%$ downregulated.
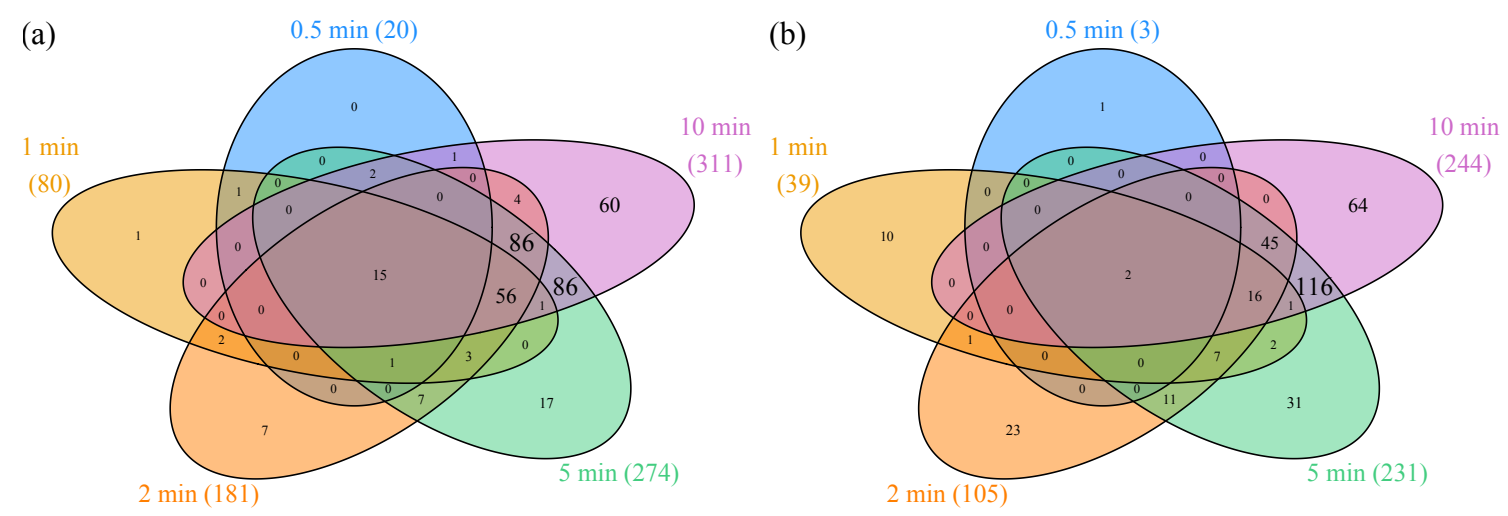

Figure 1. Venn diagrams of differentially expressed (DE) genes. Number of genes with increasing (a) and decreasing (b) expression compared to anaerobic control at the specified time points (total number of DE genes at the specified time points are provided in brackets). The numbers in overlapping regions represent $\mathrm{DE}$ genes at the corresponding time points. Areas are not drawn to scale. Genes are considered DE compared to anaerobic steady state, if $\mid$ fold change (FC) $\mid>2$ and false discovery rate (FDR) corrected $p$ value $<0.05$.

The total number of genes that are DE at any time point between 0 and $10 \mathrm{~min}$ is 678 , out of 4295 mapped genes. This number exceeds the number of DE genes at $10 \mathrm{~min}$ ( 555 genes), so a set of genes exists that is transiently up- or downregulated. Genes that exert significant transient expression patterns lie outside the 10-min ellipses of the Venn diagrams (Figure 1).

In order to get an overview of the transient pattern of differential expression, a heatmap of logFC values was generated (Figure S2). Genes that are DE in at least one timepoint are depicted. Genes are grouped according to transient up- or downregulation and subsequently sorted according to the FC in the $10 \mathrm{~min}$ sample. The heatmap indicates that almost half of the genes with differential expression exhibit a transient expression pattern (310 out of 678 genes or 45.7\%; areas 2 and 3 in Figure S2). 


\subsubsection{Principal Component Analysis}

PCA is used to structure large datasets and to find the most characteristic expression patterns amongst genes. For PCA, regularized logarithm transformed counts were used in order not to overestimate changes in very high or low expressed genes [31]. The first two principal components cover about $74 \%$ of the variance in the data (see scree plot in Figure S3). The loadings of PC1 and PC2 are plotted in Figure S4 (genes can be identified by pointing at the dots). The time courses of the three most positive and negative genes of PC1 and PC2 are provided in Figures 2 and 3, respectively. The genes shown in Figure 2a-c have a positive loading in PC1. Expression of these genes is increasing with time. Figure $2 \mathrm{~d}-\mathrm{f}$ shows genes with negative loading and here expression decreases. Therefore, the feature increasing or decreasing expression is identified by the PCA describing most variance $(63 \%)$. For PC2, all displayed genes (Figure $3 a-c$ with positive loading, $d-f$ with negative loading) exert an increasing expression. However, positively loaded genes are increasing predominantly between $2 \mathrm{~min}$ and $10 \mathrm{~min}$, while negatively loaded genes increase between $0.5 \mathrm{~min}$ and $2 \mathrm{~min}$. This feature-early or late responding genes-is the second important property detected by PCA ( $11 \%$ variance).

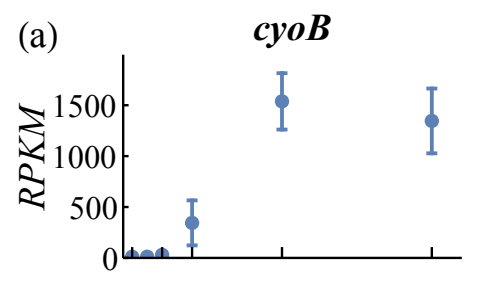

(b)

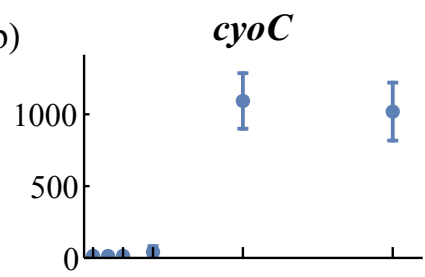

(e)

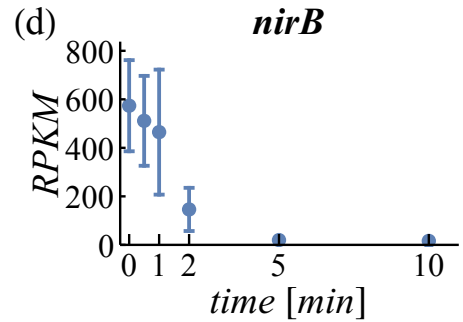

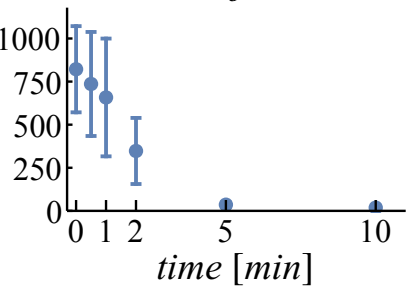

(c)

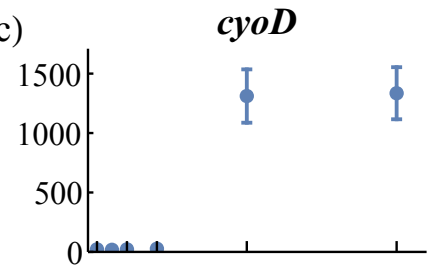

(f)

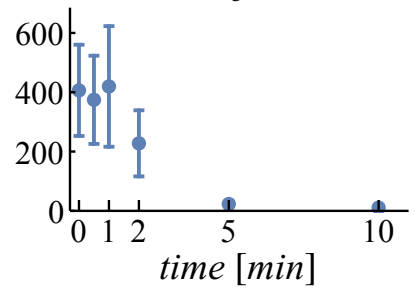

Figure 2. Loadings of principal component 1. Most positively loaded genes are given in the top row $(\mathbf{a}-\mathbf{c})$, negatively loaded genes in the bottom row $(\mathbf{d}-\mathbf{f})$. RPKM: reads per kilobase per million reads.

(a)

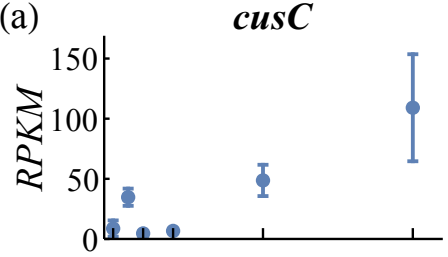

(d)

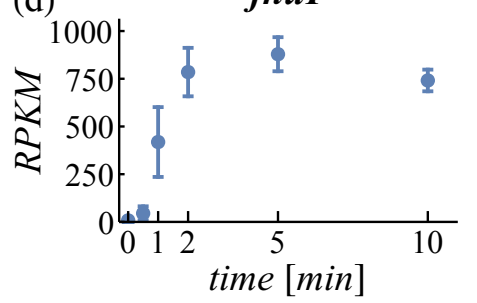

(b)

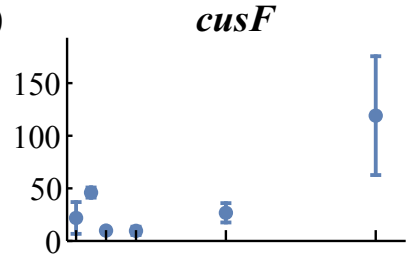

(e)

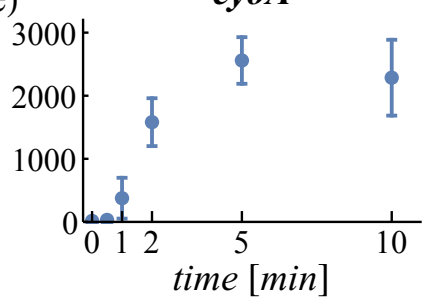

(c)

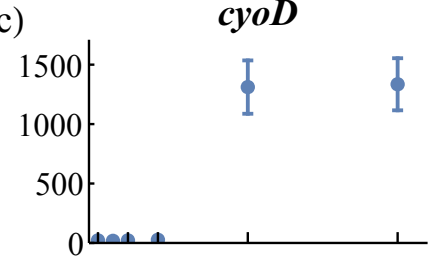

(f)

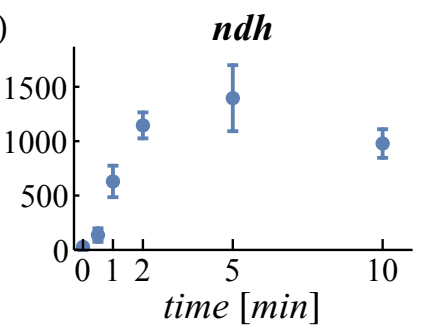

Figure 3. Loadings of principal component 2. Most positively loaded genes are given in the top row $(\mathbf{a}-\mathbf{c})$, negatively loaded genes in the bottom row $(\mathbf{d}-\mathbf{f})$. 


\subsubsection{Regulon and Gene Ontology Enrichments}

To classify the up- and downregulated genes on the functional level, a regulon and GO enrichment analysis was carried out using Pathway Tools [33,34]. Significant enrichments of a selection of regulators and GO terms are plotted in Figure 4a for upregulated genes and in Figure 4b for downregulated genes (the full list of enrichments is available from Figure S5 and Table S3 detailing the matched genes).

(a)

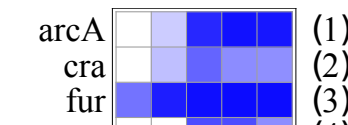

GO:0006091 - generation of precursor metabolites and energy

GO:0006099 - tricarboxylic acid cycle GO:0006598 - polyamine catabolic process $\mathrm{GO}: 0006826$ - iron ion transport

GO:0006879 - cellular iron ion homeostasis

GO:0006979 - response to oxidative stress GO:0009060 - aerobic respiration

GO:0009239 - enterobactin biosynthetic process GO:0015688 - iron chelate transport

0015980 - energy derivation by oxidation of organic compounds GO:0016226 - iron- sulfur cluster assembly GO:0045333 - cellular respiration GO:0055072 - iron ion homeostasis GO:0055114 - oxidation- reduction process GO:0097428 - protein maturation by iron- sulfur cluster transfer

(b)

GO:0006066 - alcohol metabolic process arcA
cra
fnr
narL
rocess
energy GO:0006091 - generation of precursor metabolites and energy
GO:0006113 - fermentation GO:0006113 - fermentation GO:0009061 - anaerobic respiration 0015980 - energy derivation by oxidation of organic compounds GO:0022904 - respiratory electron transport chain GO:0032445 - fructose import GO:0042126 - nitrate metabolic process

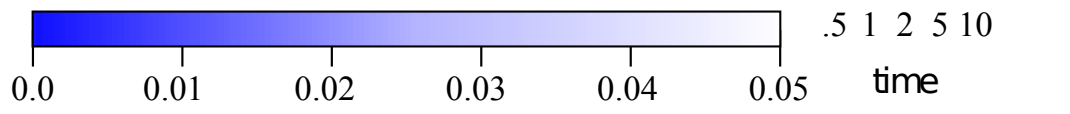

$\begin{array}{lcccccc}0.0 & 0.01 & 0.02 & 0.03 & 0.04 & 0.05 & \text { time }\end{array}$

Figure 4. Selection of regulon and gene ontology (GO) enrichments of upregulated (a) and downregulated (b) genes compared to anaerobic state. Full list is available from Figure S5. $p$ values with Bonferroni correction of the enrichments are represented.

The ferric-uptake regulator Fur is the only enriched class at $0.5 \mathrm{~min}$. Its targets remain enriched for the 10-min course and have overlaps with classes of iron homeostasis as well as metabolic classes (tricarboxylic acid, TCA) and electron transport chain (ETC), Table S3). Targets of ArcA and Cra are enriched both in upregulated and downregulated sets. Another global aerobic regulator, FNR, is only enriched in the downregulated gene set.

GO classes related to iron homeostasis and iron transport predominate the list of upregulated genes (Figure 4a, lines 7, 8, 11, 12, 14, 16, 18). The other GO classes are related to aerobic metabolism, especially the TCA cycle (line 5), and aerobic respiration (lines 10, 13, 15, and 17). Upregulation of these groups appears to initiate later compared to iron homeostasis genes (at 2 or $5 \mathrm{~min}$ ). Furthermore, genes linked to the generation of polyamines are overrepresented, especially in the 5- and 10-min samples (line 6).

Significantly overrepresented GO classes among downregulated genes can only be detected in the 5- and 10-min samples (Figure 4b). Gene sets associated with anaerobic electron transport 
predominate here (lines 8, 10, and 11). These classes overlap with the regulon of NarL (line 4). Even though no nitrate was present in the growth medium (Table S1), cells seem to express nitrate respiration genes just in case. The metabolic group of fermentative genes is only overrepresented in the 5- and 10-min samples (line 7) and can, therefore, be assigned to late responding genes.

The GO classes "generation of precursor metabolites and energy" and "energy derivation by oxidation of organic compounds" contain both aerobic as well as anaerobic genes. The classes are overrepresented in both GO analyses (Figure 4a, lines 4 and 13; Figure 4b, lines 6 and 9).

\subsection{Transcriptional Elongation Rates}

TERs and transcriptional delay times of individual operons were determined by estimating the times at which expression of successive subsections of an operon begin to increase or decrease (Figure 5a,b). The average TER obtained does not account for possible fluctuations of the elongation speed. For readability, we will stick to the term TER instead of average TER throughout this article, bearing in mind that fluctuations may occur. Our expression data show both decreasing and increasing expression. In the former case, the rate of the last polymerase bound before the promoter became less active is determined. In the latter case, the rate of the first polymerase after promoter activation is tracked.

(a)

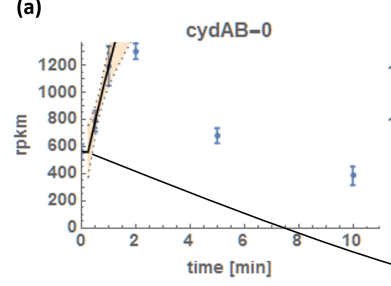

(c)

Elongation rates [nt/s]

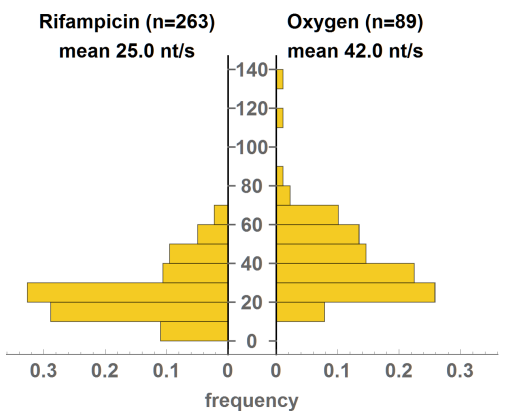

cydAB-4
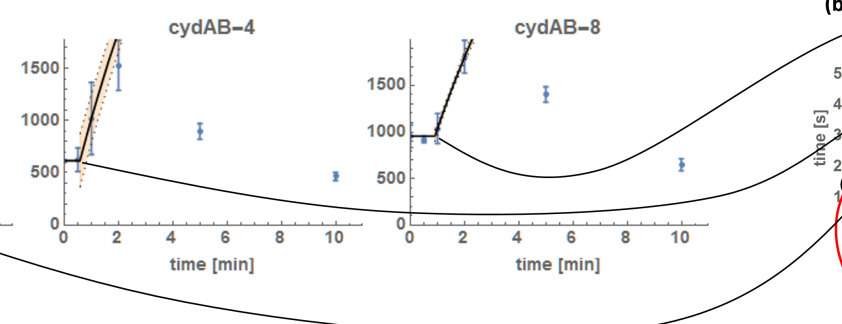

(b)
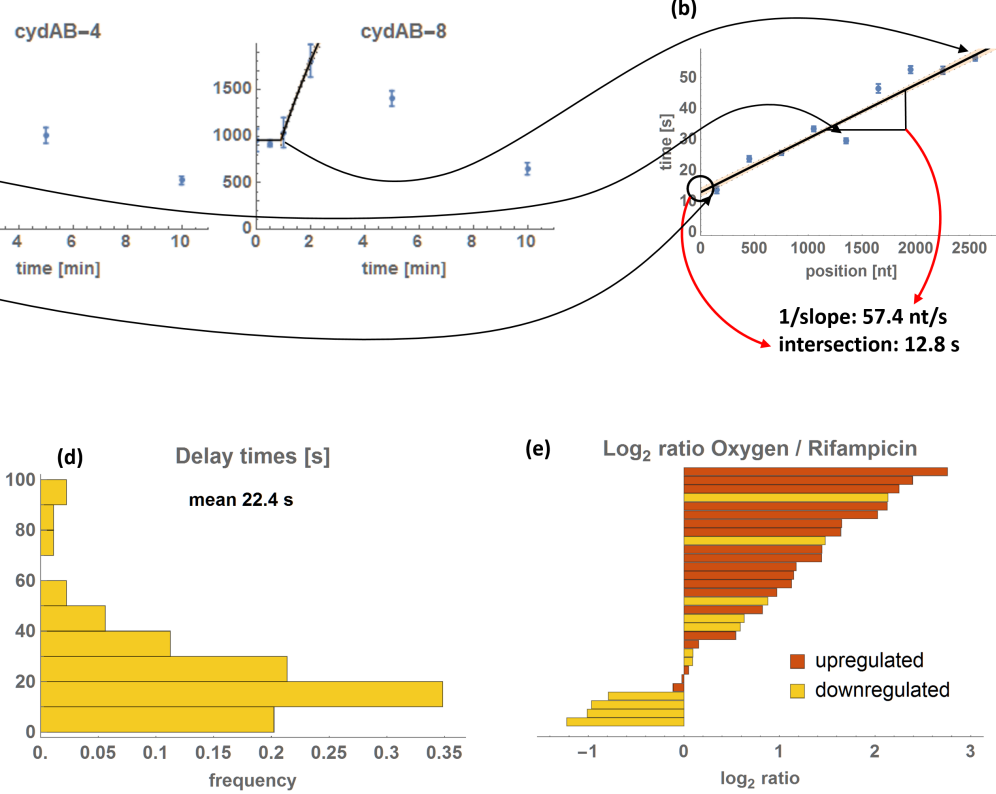

(e) $\log _{2}$ ratio

Figure 5. Calculation and overview of transcriptional elongation rates (TERs). (a) The calculation method is illustrated with the example of the $c y d A B$-operon. Depicted is the expression of the 0th (1-300 nt), 4th (1201-1500 nt), and 8th (2401-2700 nt) bin of the operon (in RPKM \pm standard deviation) together with the ordinary differential equation fit of the data. (b) The time points of the kinks are plotted over the positions of the respective bins. The inverse slope of a linear regression of these data gives the TER of the operon, the intersection with the $y$-axis (position 0 of the operon) gives the delay of transcription initiation. (c) Histogram of the resultant TERs compared to those obtained by Chen et al. [13]. (d) Histogram of the obtained transcription delay times. (e) Comparison of the TERs of individual operons obtained by oxygen and rifampicin methods; $\log _{2}$ ratios are indicated together with upregulation (red) or downregulation (orange) of the oxygen treatment group. Detailed data are available in Table S4.

We were able to infer TERs for 89 operons with a median TER of $38.6 \mathrm{nt} / \mathrm{s}$ with the $10 \%$ quantile at $21.5 \mathrm{nt} / \mathrm{s}$ and the $90 \%$ quantile at $63.6 \mathrm{nt} / \mathrm{s}$. The delay times of transcription initiation have a median of $17.9 \mathrm{~s}$ with the $10 \%$ and $90 \%$ quantiles at $4.0 \mathrm{~s}$ and $43.0 \mathrm{~s}$, respectively (Figure $5 \mathrm{c}, \mathrm{d}$, Table S4). 
In few cases, negative delay times were estimated by linear regression and in one case $(a d h E)$ the $95 \%$ confidence interval does not include the zero. Those values are relatively small (up to $-7 \mathrm{~s}$ ) and can be assigned to variations of TERs within the operons (higher initial TER). In the histogram of delay times (provided in Figure 5d) the negative values have been changed to zero, indicating immediate expression.

\subsubsection{Correlation Analyses of TERs and Delay Times}

What are the driving forces governing TERs? We approached this question by evaluating Spearman correlations of the obtained TERs with characteristics of the respective operons. Table 1 gives a summation of our findings (details available in Table S4). The CAI is a measure for the codon usage bias [38]. In our data, the estimated TERs negatively correlate with CAI (Table 1). The same holds for the more recent ITE [40]). All other correlations concerning expression levels, sequence features, sigma factors, and regulators were not significant ( $p$ value $>0.05)$.

Table 1. Spearman correlations $(\rho)$ of TERs and delay times with expression levels, sequence features, regulators, and sigma factors.

\begin{tabular}{ccccc}
\hline & $\boldsymbol{\rho}$ of TER & $\boldsymbol{p}$ Value & $\boldsymbol{\rho}$ of Delay Time & $\boldsymbol{p}$ Value \\
\hline upregulation & -0.084 & 0.43 & 0.37 & $\mathbf{2 . 7} \times \mathbf{1 0}^{-\mathbf{4}}$ \\
maximal FC & -0.17 & 0.10 & 0.416 & $\mathbf{7 . 2} \times \mathbf{1 0}^{-\mathbf{6}}$ \\
maximal RPKM & -0.10 & 0.36 & 0.05 & 0.61 \\
GC content & -0.11 & 0.32 & -0.04 & 0.73 \\
CAI & -0.23 & $\mathbf{0 . 0 3}$ & -0.10 & 0.35 \\
ITE & -0.25 & $\mathbf{0 . 0 2}$ & -0.15 & 0.14 \\
RNA folding energy & 0.17 & 0.10 & 0.12 & 0.25 \\
regulated by Cra * & -0.03 & 0.75 & -0.29 & $\mathbf{0 . 0 1}$ \\
regulated by FNR * & -0.13 & 0.21 & -0.21 & $\mathbf{0 . 0 4}$ \\
regulated by Fur-Fe * & -0.12 & 0.28 & 0.22 & $\mathbf{0 . 0 4}$ \\
controlled by sigma S & -0.15 & 0.15 & -0.22 & $\mathbf{0 . 0 4}$ \\
\hline
\end{tabular}

* without differentiation between activation or repression. Values in bold indicate significant correlation $(p$ value $<0.05)$. CAI: codon adaptation index; FC: fold change; ITE: index of translation elongation; RPKM: reads per kilobase per million reads; TER: transcription elongation rate.

Upregulation, as a binary feature, as well as high maximal FC, correlates positively with delay times. Contributions of the sigma factor $S$, and of the regulators Cra and FNR are correlated with short delay times. Conversely, the regulator Fur-Fe correlates with long delay times. Other regulators, as well as the number of regulators involved in the regulation of the respective operon, have no significant influence on the delay time (Table S4).

\section{Discussion}

\subsection{Gene Expression Patterns}

Cellular response to an aerobic shift can be characterized by early ( 0 to $1 \mathrm{~min}$ ) and late ( 5 to $10 \mathrm{~min}$ ) altered gene expression (Figure 3). Significant differential expression of 23 genes within 30 s (Table S2) indicates immediate cell adaptation to the changed environment. The early sampling points at 0.5 and 1 min enable the study of the order in which differential gene expression appears. As most of the dynamic changes take place within the initial 5 min after the switch, our dataset is much richer in detail compared to earlier studies [4,5] where the first sampling points are at $5 \mathrm{~min}$ and $2 \mathrm{~min}$, respectively. The 2-min sampling point in Rolfe et al. [5] is sufficient to grasp also transient upregulation, such as in $c y d A B$, which is missed in the Partridge et al. data [4]. However, the earlier time points are required to follow the increase rather than only the decrease in expression.

The total number of both upregulated and downregulated genes increases steadily during the initial $10 \mathrm{~min}$ after aerobic shift to 555 genes (Figure 1). However, the composition of DE genes 
is not just increasing, but rather changing with time. Besides early and late cell response, gene expression data reveal transient up- or downregulation (e.g., Figure 3f) as a common characteristic (Figure S2). Several reasons can be conceived as to why transient expression could be advantageous for the cell: (1) expression of stress genes, as the stress from oxygen is only present until the cell has adapted to it; (2) expression of enzymes that are optimal under microaerobic conditions. Microaerobic conditions occur right after the initiation of aeration, however, only for a few seconds, as $100 \%$ aerobiosis is reached at a $\mathrm{pO}_{2}$ between 0.5 and $5 \%$ when oxidases are saturated (Figure $\mathrm{S} 1[5,44]$ ); (3) by temporarily overexpressing the transcript of a required enzyme, total translated amounts of the respective protein will be higher, i.e., in the competition for ribosomes, the more abundant mRNAs will be translated proportionally [45]; (4) transient need for alternative metabolic enzymes due to capacity constraints $[8,46]$. The capacity constraints arise from slow gene expression, or from inactivation of the preferred enzymes. Alternative enzymes are often enzymatically less efficient (i.e., more enzymatic transformations are needed for the same net reaction) or metabolically less efficient (i.e., less energy is conserved from the reaction; this classification refers to parsimonious enzyme usage flux balance analysis [12]). Not surprisingly, PCA detected the distinction of increasing or decreasing expression as the feature that accounts for most variance (Figure S3). The second property determined by PCA was early or late differential expression. This depends on the time needed for regulation and on the position of the gene within the operon (i.e., the distance from the promoter). In the regulon and GO enrichment analysis, the iron regulator Fur, together with GO classes iron transport and homeostasis (Figure 4a, lines 3, 7, and 16), turned out to be the earliest responding classes. In the following sections, we will discuss the expression of iron homeostasis genes and aerobic respiration genes in more detail.

\subsubsection{Iron Balance}

The activity of many redox enzymes depends on the ability to oxidize ferrous iron $\left(\mathrm{Fe}^{2+}\right)$ to ferric iron $\left(\mathrm{Fe}^{3+}\right)$ in the presence of oxygen. Oxidation of $\mathrm{Fe}^{2+}$ poses stress on the cells through the inadvertent release of ROS in the Fenton reaction [47]. To encounter emerging ROS, cells maintain intracellular iron homeostasis by adapting iron uptake and storage $[47,48]$. Previous studies have suggested the emergence of a burst of ROS stress upon the transition to aerobic conditions which was deduced from the transient expression of stress genes, such as ahpCF (an OxyR target) or increased activity of the regulator OxyR [4,5]. Transient upregulation of $a h p C F$ is also evident from our data (Table S2) which leads us to the reasonable assumption of oxidative stress. The GO class "response to oxidative stress", containing 88 genes, is enriched in the 5- and 10-min samples, though there are indications that this stress is present earlier (e.g., ahpCF is upregulated starting at $1 \mathrm{~min}$ ).

The cells react to the oxidative stress by fast expression of iron homeostatic genes (Figure 4). This is also evident from the differential expression of bfd which holds the highest FC at $30 \mathrm{~s}$ after the aerobic shift (Table S2). Bfd, an iron-sulfur (FeS) cluster containing ferredoxin, is involved in iron storage and reveals the importance of iron homeostasis caused by an aerobic shift [49,50].

At the same time, the aerobic shift induces expression of ETC genes, whose products require $\mathrm{Fe}^{2+}$ for their activity (Figures $2 \mathrm{a}-\mathrm{c}$ and $3 \mathrm{c}, \mathrm{e}$ ). In turn, this activity contributes to reducing oxidative stress by facilitating a controlled reduction of molecular oxygen [48]. Consequently, cells dynamically regulate iron homeostasis according to its demand.

\subsubsection{Expression of Oxidases}

In response to an aerobic shift, $E$. coli adapts the expression of its respiratory enzymes cytochrome bo oxidase (encoded by $c y 0 A B C D$, Figures $2 \mathrm{a}-\mathrm{c}$ and $3 \mathrm{e}$ ) and cytochrome bd-I oxidase (encoded by $c y d A B$, Figure $5 \mathrm{a}$ ). Functional cytochrome bo oxidase contains ferrous iron bound to a heme group. The genes $c y o A$ and $c y o D$ were identified by PCA as representatives for early and late upregulation (Figure 3c,e), respectively, which can be ascribed to the positions of the genes within the cyo-operon. In contrast to the cyo-operon, expression of cyd-genes is increased even earlier (13-s versus 17-s delay, Table S4) and is only transient. Expression of Cyd, the main oxidase under microaerobic 
conditions, is potentially triggered by the short microaerobic phase after onset of aeration [51]. Considering its high $\mathrm{O}_{2}$ affinity, the oxygen-scavenging enzyme Cyd facilitates $\mathrm{O}_{2}$ sensing of the ArcBA two-component system by oxidizing the quinol pool even in microaerobiosis [48,52-54]. Cyd is less efficient in terms of energy conservation than Cyo. Hence, low expression levels of Cyd and high levels of Cyo under long-term aerobic conditions are beneficial for the cell. During the aerobic shift, both oxidases cooperatively reduce oxidative stress and contribute to the dynamic adaptation to the changed environment.

\subsection{Transcriptional Elongation Rates}

Using oxygen, a naturally occurring stimulus, essential transcriptional dynamics of the cellular response were investigated on a short time scale. Our obtained TER values are in good agreement with earlier reports that used pulse chase or hybridization techniques for TER estimation $[55,56]$. We compared our TER results to Chen et al. [13], who used rifampicin to block transcription initiation. Rifampicin binds free RNA polymerases irreversibly and globally prevents transcription initiation [57]. 30 of our analyzed operons are contained in the results of Chen et al. (Figure 5e).

In general, the mean TER was higher after oxygen treatment than after rifampicin treatment (Figure 5c). The ratios of $\mathrm{TER}_{\text {oxygen }} / \mathrm{TER}_{\text {rifampicin }}$ range from halved rate to no difference to 7 -fold increase (Figure 5e). High TER $\mathrm{oxygen}_{\mathrm{TER}}$ rifampicin ratios are correlated with an upregulation in the oxygen treatment group (while after rifampicin treatment, all operons are downregulated). The difference between the measurement of upregulated and downregulated operons is that at downregulation, the operon sequence is initially loaded with RNA polymerases. Simplified, as soon as the promoter is turned off, the position of the endmost polymerase can be tracked. Conversely, at upregulation, the operon sequence is empty initially and the position of the first polymerase binding after the promoter is switched on can be followed. It has been shown that subsequent polymerases are able to push the preceding polymerase over roadblocks and prevent proofreading, thereby accelerating transcription $[15,58]$. This is not the case for the polymerase that is last in line, whose position is determined in operons with decreasing expression. This effect could explain the strong association of upregulated operons with increased TER compared to rifampicin (Figure 5e). The mean TER was higher in our study $(42.0 \mathrm{nt} / \mathrm{s})$ compared to Chen et al. $(25.0 \mathrm{nt} / \mathrm{s}$, Figure $5 \mathrm{c})$. This might be a result of the high representation of upregulated operons (62 of 89).

Otherwise, upregulation does not generally correlate with TER, indicating that other factors are more pivotal for TER. In addition, the maximal FC or maximal RPKM, indicators for strong promoters, do not correlate with fast TERs, even though a high polymerase density is likely present (Table 1). We did not expect this, because in the case of upregulation, fast TERs might contribute to increase transcript levels and vice versa for downregulated operons. However, the opposite seems to be the case. The strong correlation between delay times and maximal FCs suggests that strong induction results in delayed polymerase binding. However, high FCs might also (at least in part) be attributed to differences in transcript stability rather than synthesis.

Concerning sequence features, the GC content, or any other nucleotide content (Table S4), was irrelevant for TER, even though more hairpin loops are formed in GC-rich sequences. This result is consistent with the finding of Proshkin et al. [15], that hairpins are not responsible for TER decrease. CAI is a measure of codon usage bias of a sequence and high CAI values are associated with fast translation rates [38,59-61]. Contrary to our data (Table S4), a positive correlation between CAI and TER was suggested, assuming cotranscriptional interactions between ribosomes and RNA polymerases $[15,16]$. We found a significant negative correlation between CAI and TER which argues against the stimulation of TER by ribosomes during cotranscriptional translation. This means that common codons are transcribed slower than rare codons and contradicts previous findings made on a small scale [15]. The recently developed ITE, which is claimed by the author to be a superior indicator of translation elongation, also negatively correlates with TER (Table S4). We also checked for correlation of the RNA 
folding energy with TER. The underlying idea was that the folding of the nascent RNA chain pushes the polymerase forward. We found no significant effect.

The second feature obtained from TER estimation is the delay time from stimulus until transcription sets in. This delay depends on the time required for regulators to sense the stimulus and to set up differential regulation. By comparing our data with various regulators and sigma factors we identified Cra, FNR, and sigma $\mathrm{S}$ as regulators correlated with short delay times. FNR is involved in the regulation of aerobic and anaerobic enzymes [62]. It is directly inactivated by oxygen (via oxidation of a FeS cluster [63,64]). This explains the short delay. Cra is a global regulator of carbon metabolism and influences expression of genes related to gluconeogenesis, TCA cycle, and glycolysis, among others [65]. Effectors of Cra are fructose-1-phosphate and fructose-1,6-bisphosphate which act as sensors of glycolytic flux (which is, in turn, higher in anaerobic cultures than under aerobic conditions $[66,67])$. Compared to the redox state, this effect is rather indirect. We therefore expected changes of fructose-phosphate levels to occur later than changes in the redox state of the cell, so that FNR-regulated operons were expected to have shorter delays than Cra regulated operons, which was not the case in our data. Sigma $S$ is the sigma factor responsible for general stress response [68]. For this reason, we expected a fast response of sigma S-dependent operons, which is confirmed by our data.

Genes regulated by the ferric-uptake regulator Fur-Fe are correlated with long delay times. Fur-Fe targets are mainly involved in iron homeostasis [69] and its targets were found to be enriched from the 0.5 min sample on. Fur binds to its DNA target sequences in the presence of intracellular ferrous iron $\left(\mathrm{Fe}^{2+}\right)$ thereby repressing expression of target genes. The redox potential of intracellular iron is much higher than that of iron bound to FeS clusters (FNR) or cysteines in a disulfide bond (OxyR) $[70,71]$. This can cause the long delay time of Fur-Fe depending operons. Furthermore, an upregulation of fur can be observed after the stimulus (Table S2). Varghese et al. show that upregulation of the regulator Fur through OxyR in an oxidative stress environment counteracts the inactivation via oxidation and restores repression of Fur target genes [72,73]. This mechanism prevents the early import of iron which would result in additional ROS stress through the Fenton reaction.

However, Fur targets overlap to a large extent ( $p$ value of the Spearman correlation of 0.002 , Table S4) with the feature upregulation which correlates with long delays. Ultimately, this might be the underlying cause for the longer delay of Fur targets. Cra and FNR targets correlate insignificantly with downregulation which might contribute to their observed fast initiation.

Concerning expression levels, upregulation and maximal FC correlated positively with the delay times, indicating that the cell response via upregulation takes longer than via downregulation. This effect might be assigned to the competition of promoters to RNA polymerases [74]. Since more genes are upregulated than downregulated in our experiment, more active promoters compete for the same number of polymerases and recruitment takes longer. Formation of an active transcription complex is a multistep process and several time constants apply to get from the closed initiation complex to the active open complex conformation [75]. This additional time is not required for stopping transcription (here, only binding or dissociation of the respective regulators are needed). The faster response of downregulated operons is, therefore, plausible. The two properties "upregulation" and "maximal FC" resemble each other (low values or zero for downregulation, high values or 1 for upregulation). Therefore, similar correlations were expected.

The range of total transcription times (from stimulus until completed transcription) from $30 \mathrm{~s}$ to $5 \mathrm{~min}$ is similar to the mixing times of large-scale bioreactors. An average cell travels in one mixing time through the reactor volume from an oxygen-rich to an oxygen-depleted environment and back. This means that in most cases the stimulus (here oxygen) will be gone before even transcription of an operon is finished. Yet more time is needed for translation and post-translational modifications, so the point where the response to the stimulus is completed becomes independent from the actual environment. This needs to be investigated, e.g., by applying continuing shifts in aeration to a laboratory scale culture. 
The $n d h$ operon, containing only one gene, is the fastest among upregulated operons with $34 \mathrm{~s}$ (Table S4, Figure 3f). The iron-independent Ndh catalyzes the first step in the ETC but it is metabolically less efficient than Nuo [76,77]. Ready availability, as demonstrated by the short total transcription time, is an advantage of $n d h$ and might be a reason for its upregulation (Figure $3 \mathrm{f}[8]$ ). Expression of less efficient genes might be in part responsible for yield losses observed in large scale fermentations [3,78].

Supplementary Materials: The following are available online at www.mdpi.com/2073-4425/8/3/90/s1. Figure S1: Dissolved oxygen concentration increases after the onset of aeration. Figure S2: Heatmap of logFCs of DE genes. Genes with differential expression in at least one time point were analyzed for transient expression patterns and plotted in the order of the FC at 10 min compared to anaerobic control. (1 and 2) Genes with increased expression after $10 \mathrm{~min}$; (1) without transient upregulation; (2) with transient upregulation; (3 and 4) genes with decreased expression after $10 \mathrm{~min}$; with (3) and without (4) transient downregulation. The sections 2 and 3 indicate genes with transient effects and make up about half of all DE genes. Figure S3: Scree plot of PCA. The plot shows the percentage of variance of the principal components. Figure S4: Rotation of PCA. The rotation plot indicates the contributions of all genes to the PCs 1 and 2. Gene names can be identified by pointing at the dots in the plot. Figure S5: Regulon and GO enrichments of upregulated (a) and downregulated (b) genes compared to anaerobic state. $p$ values with Bonferroni correction of the enrichments are represented. Table S1: Composition of defined medium, modified from [79]. Table S2: Differential expression: logFCs are provided together with expression levels and $p$ values for differential expression for all genes analyzed. Table S3: Regulon and GO enrichment analyses. $p$ values and matched genes for every class are indicated. Table S4: TERs and delay times of analyzed operons. Pearson and Spearman correlations with respective $p$ values are provided for TER and delay times after aerobic shift.

Acknowledgments: The authors thank the whole "RecogNice" project group for productive collaboration and fruitful discussions: Ralf Takors, Michael Löffler, and Joana Simen (Institute of Biochemical Engineering, University of Stuttgart), Michael Ederer, Julia Lischke, and Samantha Kunz (Institute for System Dynamics, University of Stuttgart), Olaf Rieß and Karin Schäferhoff (Institute of Medical Genetics and Applied Genomics, University of Tübingen), and Georg Sprenger and Natalia Trachtmann (Institute of Microbiology, University of Stuttgart). The authors further gratefully acknowledge the funding of this work by the Bundesministerium für Bildung und Forschung (BMBF Grant FKZ0316178A).

Author Contributions: J.W., O.S. and R.F. conceived and designed the experiments; J.W. performed the experiments; J.W., A.U. and G.J. analyzed the data; J.W. drafted the manuscript; A.U., O.S. and R.F. revised the manuscript.

Conflicts of Interest: The authors declare no conflict of interest. The funding sponsors had no role in the design of the study; in the collection, analyses, or interpretation of data; in the writing of the manuscript, and in the decision to publish the results.

\section{References}

1. Junker, B.H. Scale-Up Methodologies for Escherichia coli and Yeast Fermentation Processes. J. Biosci. Bioeng. 2004, 97, 347-364.

2. Takors, R. Scale-up of microbial processes: Impacts, tools and open questions. J. Biotechnol. 2012, 160, 3-9.

3. Lara, A.R.; Galindo, E.; Ramírez, O.T.; Palomares, L.A. Living With Heterogeneities in Bioreactors: Understanding the Effects of Environmental Gradients on Cells. Mol. Biotechnol. 2006, 34, 355-382.

4. Partridge, J.D.; Scott, C.; Tang, Y.; Poole, R.K.; Green, J. Escherichia coli Transcriptome Dynamics during the Transition from Anaerobic to Aerobic Conditions. J. Biol. Chem. 2006, 281, 27806-27815.

5. Rolfe, M.D.; Ocone, A.; Stapleton, M.R.; Hall, S.; Trotter, E.W.; Poole, R.K.; Sanguinetti, G.; Green, J. Systems analysis of transcription factor activities in environments with stable and dynamic oxygen concentrations. Open Biol. 2012, 2, 120091.

6. Trotter, E.W.; Rolfe, M.D.; Hounslow, A.M.; Craven, C.J.; Williamson, M.P.; Sanguinetti, G.; Poole, R.K.; Green, J. Reprogramming of Escherichia coli K-12 Metabolism during the Initial Phase of Transition from an Anaerobic to a Micro-Aerobic Environment. PLoS ONE 2011, 6, e25501.

7. Ederer, M.; Steinsiek, S.; Stagge, S.; Rolfe, M.D.; Ter Beek, A.; Knies, D.; Teixeira de Mattos, M.J.; Sauter, T.; Green, J.; Poole, R.K.; et al. A mathematical model of metabolism and regulation provides a systems-level view of how Escherichia coli responds to oxygen. Front. Microbiol. 2014, 5, 124.

8. von Wulffen, J.; Sawodny, O.; Feuer, R. Transition of an Anaerobic Escherichia coli Culture to Aerobiosis: Balancing mRNA and Protein Levels in a Demand-Directed Dynamic Flux Balance Analysis. PLoS ONE 2016, 11, e0158711. 
9. Bettenbrock, K.; Bai, H.; Ederer, M.; Green, J.; Hellingwerf, K.J.; Holcombe, M.; Kunz, S.; Rolfe, M.D.; Sanguinetti, G.; Sawodny, O.; et al. Towards a Systems Level Understanding of the Oxygen Response of Escherichia coli. Adv. Microb. Physiol. 2014, 64, 65-114.

10. Löffler, M.; Simen, J.D.; Jäger, G.; Schäferhoff, K.; Freund, A.; Takors, R. Engineering E. coli for Large-Scale Production-Strategies Considering ATP Expenses and Transcriptional Responses. Metab. Eng. 2016, 38, 73-85.

11. Kaleta, C.; Schäuble, S.; Rinas, U.; Schuster, S. Metabolic costs of amino acid and protein production in Escherichia coli. Biotechnol. J. 2013, 8, 1105-1114.

12. Lewis, N.E.; Hixson, K.K.; Conrad, T.M.; Lerman, J.A.; Charusanti, P.; Polpitiya, A.D.; Adkins, J.N.; Schramm, G.; Purvine, S.O.; Lopez-Ferrer, D.; et al. Omic data from evolved E. coli are consistent with computed optimal growth from genome-scale models. Mol. Syst. Biol. 2010, 6, 390.

13. Chen, H.; Shiroguchi, K.; Ge, H.; Xie, X.S. Genome-wide study of mRNA degradation and transcript elongation in Escherichia coli. Mol. Syst. Biol. 2015, 11, 781.

14. Miller, O.L.; Hamkalo, B.; Thomas, C. Visualization of bacterial genes in action. Science 1970, 169, $392-395$.

15. Proshkin, S.; Rahmouni, A.R.; Mironov, A.; Nudler, E. Cooperation Between Translating Ribosomes and RNA Polymerase in Transcription Elongation. Science 2010, 328, 504-508.

16. Burmann, B.M.; Schweimer, K.; Luo, X.; Wahl, M.C.; Stitt, B.L.; Gottesman, M.E.; Rösch, P. A NusE:NusG complex links transcription and translation. Science 2010, 328, 501-504.

17. Morgan, E.A. Antitermination mechanisms in rRNA operons of Escherichia coli. J. Bacteriol. 1986, 168, 1-5.

18. Rutberg, B. Antitermination of transcription of catabolic operons. Mol. Microbiol. 1997, 23, 413-421.

19. Washburn, R.S.; Gottesman, M.E. Regulation of transcription elongation and termination. Biomolecules 2015, 5, 1063-1078.

20. Jahn, S.; Haverkorn van Rijsewijk, B.R.; Sauer, U.; Bettenbrock, K. A role for EIIA ${ }^{N t r}$ in controlling fluxes in the central metabolism of E. coli K12. Biochim. Biophys. Acta 2013, 1833, 2879-2889.

21. Zeppenfeld, T.; Larisch, C.; Lengeler, J.W.; Jahreis, K. Glucose Transporter Mutants of Escherichia coli K-12 with Changes in Substrate Recognition of $\mathrm{IICB}^{G l c}$ and Induction Behavior of the ptsG Gene. J. Bacteriol. 2000, $182,4443-4452$.

22. Andrews, S. FastQC: A Quality Control Tool for High Throughput Sequence Data. Available online: http://www.bioinformatics.babraham.ac.uk/projects/fastqc/ (accessed on 26 February 2015).

23. Schneider, K.L.; Pollard, K.S.; Baertsch, R.; Pohl, A.; Lowe, T.M. The UCSC Archaeal Genome Browser. Nucleic Acids Res. 2006, 34, D407-D410.

24. Dobin, A.; Davis, C.A.; Schlesinger, F.; Drenkow, J.; Zaleski, C.; Jha, S.; Batut, P.; Chaisson, M.; Gingeras, T.R. STAR: Ultrafast universal RNA-seq aligner. Bioinformatics 2013, 29, 15-21.

25. Anders, S.; Pyl, P.T.; Huber, W. HTSeq-A Python framework to work with high-throughput sequencing data. Bioinformatics 2015, 31, 166-169.

26. Edgar, R.; Domrachev, M.; Lash, A.E. Gene Expression Omnibus: NCBI gene expression and hybridization array data repository. Nucleic Acids Res. 2002, 30, 207-210.

27. Robinson, M.D.; McCarthy, D.J.; Smyth, G.K. edgeR: A Bioconductor package for differential expression analysis of digital gene expression data. Bioinformatics 2010, 26, 139-140.

28. R Development Core Team. R: A Language and Environment for Statistical Computing; R Foundation for Statistical Computing: Vienna, Austria, 2014.

29. Benjamini, Y.; Hochberg, Y. Controlling the false discovery rate: A practical and powerful approach to multiple testing. J. R. Stat. Soc. 1995, 57, 289-300.

30. Chen, H.; Boutros, P.C. VennDiagram: A package for the generation of highly-customizable Venn and Euler diagrams in R. BMC Bioinform. 2011, 12, 35.

31. Love, M.I.; Huber, W.; Anders, S. Moderated estimation of fold change and dispersion for RNA-seq data with DESeq2. Genome Biol. 2014, 15, 1-34.

32. Mardia, K.V.; Kent, J.T.; Bibby, J.M. Multivariate Analysis; Academic Press: New York, NY, USA, 1979.

33. Karp, P.D.; Paley, S.; Romero, P. The Pathway Tools software. Bioinformatics 2002, 18, S225-S232.

34. Karp, P.D.; Paley, S.M.; Krummenacker, M.; Latendresse, M.; Dale, J.M.; Lee, T.J.; Kaipa, P.; Gilham, F.; Spaulding, A.; Popescu, L.; et al. Pathway Tools version 19.0: Integrated Software for Pathway/Genome Informatics and Systems Biology. Brief. Bioinform. 2010, 11, 1-14. 
35. Lawrence, M.; Huber, W.; Pagès, H.; Aboyoun, P.; Carlson, M.; Gentleman, R.; Morgan, M.T.; Carey, V.J. Software for Computing and Annotating Genomic Ranges. PLoS Comput. Biol. 2013, 9, e1003118.

36. Bernstein, J.A.; Khodursky, A.B.; Lin, P.H.; Lin-Chao, S.; Cohen, S.N. Global analysis of mRNA decay and abundance in Escherichia coli at single-gene resolution using two-color fluorescent DNA microarrays. Proc. Natl. Acad. Sci. USA 2002, 99, 9697-9702.

37. Kirkpatrick, S.; Gelatt, C.D.; Vecchi, M.P. Optimization by simulated annealing. Science 1983, 220, 671-680.

38. Sharp, P.M.; Li, W.H. The codon adaptation index-A measure of directional synonymous codon usage bias, and its potential applications. Nucleic Acids Res. 1987, 15, 1281-1295.

39. Nakamura, Y.; Gojobori, T.; Ikemura, T. Codon usage tabulated from the international DNA sequence databases; its status 1999. Nucleic Acids Res. 1999, 27, 292.

40. Xia, X. A major controversy in codon-anticodon adaptation resolved by a new codon usage index. Genetics 2015, 199, 573-579.

41. Hofacker, I.L.; Fontana, W.; Stadler, P.F.; Bonhoeffer, L.S.; Tacker, M.; Schuster, P. Fast folding and comparison of RNA secondary structures. Monatshefte Chem. Chem. Mon. 1994, 125, 167-188.

42. Lorenz, R.; Bernhart, S.H.; Höner zu Siederdissen, C.; Tafer, H.; Flamm, C.; Stadler, P.F.; Hofacker, I.L. ViennaRNA Package 2.0. Algorithms Mol. Biol. 2011, 6, 26.

43. Keseler, I.M.; Mackie, A.; Peralta-Gil, M.; Santos-Zavaleta, A.; Gama-Castro, S.; Bonavides-Martinez, C.; Fulcher, C.; Huerta, A.M.; Kothari, A.; Krummenacker, M.; et al. EcoCyc: Fusing model organism databases with systems biology. Nucleic Acids Res. 2013, 41, D605-D612.

44. Alexeeva, S.; Hellingwerf, K.J.; Teixeira de Mattos, M.J.; Teixeira de, M.J. Quantitative Assessment of Oxygen Availability: Perceived Aerobiosis and Its Effect on Flux Distribution in the Respiratory Chain of Escherichia coli. J. Bacteriol. 2002, 184, 1402-1406.

45. De Vos, D.; Bruggeman, F.J.; Westerhoff, H.V.; Bakker, B.M. How molecular competition influences fluxes in gene expression networks. PLOS ONE 2011, 6, e28494.

46. Colijn, C.; Brandes, A.; Zucker, J.; Lun, D.S.; Weiner, B.; Farhat, M.R.; Cheng, T.Y.; Moody, D.B.; Murray, M.; Galagan, J.E. Interpreting expression data with metabolic flux models: Predicting Mycobacterium tuberculosis mycolic acid production. PLoS Comput. Biol. 2009, 5, e1000489.

47. Imlay, J.A. The molecular mechanisms and physiological consequences of oxidative stress: Lessons from a model bacterium. Nat. Rev. Microbiol. 2013, 11, 443-454.

48. Lindqvist, A.; Membrillo-Hernández, J.; Poole, R.K.; Cook, G.M. Roles of respiratory oxidases in protecting Escherichia coli K12 from oxidative stress. Antonie van Leeuwenhoek 2000, 78, 23-31.

49. Garg, R.P.; Vargo, C.J.; Cui, X.; Kurtz, D.M. A [2Fe-2S] protein encoded by an open reading frame upstream of the Escherichia coli bacterioferritin gene. Biochemistry 1996, 35, 6297-6301.

50. Harrison, P.M. Iron storage in bacteria. Nature 1979, 279, 15-16.

51. Govantes, F.; Orjalo, A.V.; Gunsalus, R.P. Interplay between three global regulatory proteins mediates oxygen regulation of the Escherichia coli cytochrome d oxidase (cydAB) operon. Mol. Microbiol. 2002, 38, 1061-1073.

52. Iuchi, S.; Chepuri, V.; Fu, H.A.; Gennis, R.B.; Lin, E.C. Requirement for terminal cytochromes in generation of the aerobic signal for the arc regulatory system in Escherichia coli: Study utilizing deletions and lac fusions of cyo and cyd. J. Bacteriol. 1990, 172, 6020-6025.

53. Iuchi, S.; Weiner, L. Cellular and molecular physiology of Escherichia coli in the adaptation to aerobic environments. J. Biochem. 1996, 120, 1055-1063.

54. Alvarez, A.F.; Rodriguez, C.; Georgellis, D. Ubiquinone and Menaquinone Electron Carriers Represent the Yin and Yang in the Redox Regulation of the ArcB Sensor Kinase. J. Bacteriol. 2013, 195, 3054-3061.

55. Bremer, H.; Yuan, D. RNA chain growth-rate in Escherichia coli. J. Mol. Biol. 1968, 38, 163-180.

56. Vogel, U.; Jensen, K.F. The RNA chain elongation rate in Escherichia coli depends on the growth rate. J. Bacteriol. 1994, 176, 2807-2813.

57. Reid, P.; Speyer, J. Rifampicin inhibition of ribonucleic acid and protein synthesis in normal and ethylenediaminetetraacetic acid-treated Escherichia coli. J. Bacteriol. 1970, 104, 376-389.

58. Epshtein, V.; Nudler, E. Cooperation between RNA polymerase molecules in transcription elongation. Science 2003, 300, 801-805.

59. Ikemura, T. Codon usage and tRNA content in unicellular and multicellular organisms. Mol. Biol. Evol. $1985,2,13-34$. 
60. Lithwick, G.; Margalit, H. Hierarchy of sequence-dependent features associated with prokaryotic translation. Genome Res. 2003, 13, 2665-2673.

61. Brockmann, R.; Beyer, A.; Heinisch, J.J.; Wilhelm, T. Posttranscriptional expression regulation: What determines translation rates? PLoS Comput. Biol. 2007, 3, 0531-0539.

62. Salmon, K.; Hung, S.p.; Mekjian, K.; Baldi, P.; Hatfield, G.W.; Gunsalus, R.P. Global Gene Expression Profiling in Escherichia coli K12: The Effects of Oxygen Availability and FNR. J. Biol. Chem. 2003, 278, 29837-29855.

63. Khoroshilova, N.; Popescu, C.; Munck, E.; Beinert, H.; Kiley, P.J. Iron-sulfur cluster disassembly in the FNR protein of Escherichia coli by $\mathrm{O}_{2}$ : [4Fe-4S] to [2Fe-2S] conversion with loss of biological activity. Proc. Natl. Acad. Sci. USA 1997, 94, 6087-6092.

64. Jervis, A.J.; Crack, J.C.; White, G.; Artymiuk, P.J.; Cheesman, M.R.; Thomson, A.J.; Le Brun, N.E.; Green, J. The $\mathrm{O}_{2}$ sensitivity of the transcription factor FNR is controlled by Ser24 modulating the kinetics of [4Fe-4S] to [2Fe-2S] conversion. Proc. Natl. Acad. Sci. USA 2009, 106, 4659-4664.

65. Ramseier, T. Cra and the control of carbon flux via metabolic pathways. Res. Microbiol. 1996, 147, 489-493.

66. Kochanowski, K.; Volkmer, B.; Gerosa, L.; Haverkorn van Rijsewijk, B.R.; Schmidt, A.; Heinemann, M. Functioning of a metabolic flux sensor in Escherichia coli. Proc. Natl. Acad. Sci. USA 2013, 110, 1130-1135.

67. Fischer, E.; Sauer, U. Metabolic flux profiling of Escherichia coli mutants in central carbon metabolism using GC-MS. Eur. J. Biochem. 2003, 270, 880-891.

68. Battesti, A.; Majdalani, N.; Gottesman, S. The RpoS-Mediated General Stress Response in Escherichia coli. Annu. Rev. Microbiol. 2011, 65, 189-213.

69. Hantke, K. Iron and metal regulation in bacteria. Curr. Opin. Microbiol. 2001, 4, 172-177.

70. Sober, H.; Loach, P. Handbook of Biochemistry, Selected Data for Molecular Biology; The Chemical Rubber Co.: Cleveland, OH, USA, 1968; pp. J27-J34.

71. Metzler, D.; Metzler, C. Biochemistry: The Chemical Reactions of Living Cells; Academic Press: New York, NY, USA, 2003; Volume 1, p. 301.

72. Varghese, S.; Wu, A.; Park, S.; Imlay, K.R.C.; Imlay, J.A. Submicromolar hydrogen peroxide disrupts the ability of Fur protein to control free-iron levels in Escherichia coli. Mol. Microbiol. 2007, 64, 822-830.

73. Mills, S.A.; Marletta, M.A. Metal Binding Characteristics and Role of Iron Oxidation in the Ferric Uptake Regulator from Escherichia coli. Biochemistry 2005, 44, 13553-13559.

74. Bremer, H.; Dennis, P.; Ehrenberg, M. Free RNA polymerase and modeling global transcription in Escherichia coli. Biochimie 2003, 85, 597-609.

75. Saecker, R.M.; Record, M.T.; Dehaseth, P.L. Mechanism of bacterial transcription initiation: RNA polymerase-Promoter binding, isomerization to initiation-competent open complexes, and initiation of RNA synthesis. J. Mol. Biol. 2011, 412, 754-771.

76. Rapisarda, V.A.; Montelongo, L.R.; Farias, R.N.; Massa, E.M. Characterization of an NADH-Linked Cupric Reductase Activity from the Escherichia coli Respiratory Chain. Arch. Biochem. Biophys. 1999, 370, 143-150.

77. Calhoun, M.W.; Gennis, R.B. Demonstration of separate genetic loci encoding distinct membrane-bound respiratory NADH dehydrogenases in Escherichia coli. J. Bacteriol. 1993, 175, 3013-3019.

78. Bylund, F.; Collet, E.; Enfors, S.O.; Larsson, G. Substrate gradient formation in the large-scale bioreactor lowers cell yield and increases by-product formation. Bioprocess Eng. 1998, 18, 171-180.

79. Hardiman, T.; Lemuth, K.; Keller, M.A.; Reuss, M.; Siemann-Herzberg, M. Topology of the global regulatory network of carbon limitation in Escherichia coli. J. Biotechnol. 2007, 132, 359-374.

(c) 2017 by the authors. Licensee MDPI, Basel, Switzerland. This article is an open access article distributed under the terms and conditions of the Creative Commons Attribution (CC BY) license (http://creativecommons.org/licenses/by/4.0/). 